Cahiers de littérature orale

\title{
La trace chantée dans la peinture kija du Kimberley oriental
}

Arnaud Morvan

\section{(2) OpenEdition}

1 Journals

\section{Édition électronique}

URL : https://journals.openedition.org/clo/916

DOI : $10.4000 /$ clo.916

ISSN : 2266-1816

Éditeur

INALCO

\section{Édition imprimée}

Date de publication : 15 janvier 2010

Pagination : 95-119

ISBN : 978-2-85831-196-5

ISSN : 0396-891X

\section{Référence électronique}

Arnaud Morvan, « La trace chantée dans la peinture kija du Kimberley oriental », Cahiers de littérature orale [En ligne], 67-68 | 2010, mis en ligne le 02 mars 2013, consulté le 30 juin 2021. URL : http:// journals.openedition.org/clo/916 ; DOI : https://doi.org/10.4000/clo.916

Ce document a été généré automatiquement le 30 juin 2021.

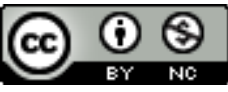

Cahiers de littérature orale est mis à disposition selon les termes de la Licence Creative Commons Attribution - Pas d'Utilisation Commerciale 4.0 International. 


\title{
La trace chantée dans la peinture kija du Kimberley oriental
}

\author{
Arnaud Morvan
}

1 L'art aborigène australien dans ses composantes rituelles ou contemporaines offre l'exemple singulier d'une tradition iconographique qui intègre des éléments d'oralité et de performance dans un processus mnémonique. Nous allons nous intéresser plus particulièrement ici à la relation entre l'image et le chant dans l'art kija du Kimberley ${ }^{1}$. En Australie, l'année 1971 marque la coïncidence de deux événements majeurs dans l'appréhension des composantes orales et picturales de la culture aborigène. Le premier est constitué par l'émergence d'un mouvement artistique aborigène qui révéla l'iconographie des groupes du désert, le second par la publication de l'ouvrage Songs of Central Australia de Theodor George Henry Strehlow.

2 L'histoire des peintres aborigènes de Papunya est désormais bien connue. Suite à l'intérêt d'un instituteur blanc, un petit groupe d'initiés de langue pintupi décide de transposer sur panneaux, puis sur toile, les motifs semi-abstraits utilisés dans les cérémonies religieuses. Des réseaux de cercles concentriques, points, demi-cercles, lignes sinueuses et empreintes animales se combinaient à l'infini pour révéler l'existence d'une cartographie mythique de l'Australie, celle des pistes de rêve ou itinéraires mythiques des ancêtres qui s'entrecroisent sur la quasi-totalité du continent. Le mouvement se répandit comme une traînée de poudre dans les autres communautés d'Australie Centrale et du Kimberley et provoqua un intérêt considérable sur la scène artistique internationale, dans les musées et chez les universitaires (Munn, 1973 ; Glowczewski, 1991b ; Dussart, 2000 ; Myers 2002).

3 Le second événement marquant cette année 1971 fut la publication du célèbre ouvrage de Theodor George Henry Strehlow Songs of Central Australia. Élevé dans la mission luthérienne d'Hermannsburg, Strehlow publiait la somme considérable de chants recueillis auprès des groupes aranda parmi lesquels il avait grandi. Pour la première fois, le chant et les formules poétiques aborigènes étaient considérés comme un objet d'étude à part entière: y étaient analysés les mesures rythmiques, la structure musicale, le vocabulaire poétique, la syntaxe, et les principales thématiques d'un large 
corpus aranda. Les chants apparaissent comme une version condensée des mythes, ne retenant que les éléments les plus saillants: noms totémiques, toponymes sacrés, actions créatrices des ancêtres. Si l'ouvrage, qui comporte des informations classifiées secrètes par les Aranda, ne fut édité qu'à cinq cents exemplaires, il constitue le socle de nombreuses études dans le domaine anthropologique, linguistique et ethnomusicologique (Barwick, 1989; Walsh, 2007). Malgré les nombreuses correspondances entre peintures et chants, aucune étude ne s'est attachée à analyser l'incidence de la parole chantée dans l'acte de création d'images. Les deux procédés semblent pourtant intrinsèquement liés dans un même dispositif mnémonique visant à enregistrer et retranscrire le parcours des ancêtres totémiques et à accéder à la mémoire contenue dans les lieux de leur passage. Dans des travaux antérieurs sur l'art kija, j'ai utilisé les concepts de trace et d'empreinte pour définir les peintures comme des représentations indiciaires d'un lieu, donnant accès à une triple mémoire mythique, historique et biographique. Reste à cerner le rôle spécifique joué par le chant dans ce dispositif. Quelles sont les modalités de la relation, notamment esthétique, entre images et chants à l'intérieur des peintures? Quels rôles jouent ces deux éléments dans la constitution d'une mémoire?

\section{Représentation indiciaire de l'espace}

4 Les peintures à l'ocre sur toile du Kimberley Oriental sont issues d'une innovation rituelle relativement récente. C'est au cours de l'année 1975, à la suite d'une série de révélations oniriques, que Rover Thomas, un exilé kukatja du désert vivant dans la communauté de Warmun ${ }^{2}$, visualisa les premières peintures sous la forme d'images, de chants et de danses transmises par l'esprit d'une parente récemment décédée. Dans le Kimberley, les nouvelles cérémonies sont souvent transmises par les esprits des morts, les djuari, lors d'expériences oniriques individuelles. Pour la plupart des groupes aborigènes, la mort inaugure un voyage au cours duquel l'esprit du défunt quitte son enveloppe charnelle pour retourner sur son site de conception où il redevient un jarrinybe (esprit-enfant), prêt à se réincarner. Pendant les rites funéraires, des chants, des danses et des peintures liées aux sites traversés doivent être exécutés sur différents supports pour permettre aux morts de rejoindre leur centre spirituel ou site de conception lié à l'espace-temps mythique Ngarrangany ou Dreaming en créole. Le terme Dreaming est utilisé par les Aborigènes à la fois pour désigner des ancêtres totémiques, les pistes de leurs voyages et l'ensemble de l'espace-temps dans lequel les hommes peuvent communiquer avec ces esprits ancestraux et forces telluriques qui sont dits dormir dans les sites sacrés et rêver la vie des hommes pour actualiser le Dreaming comme loi sacrée (Glowczewski, 1991a).

5 Les chants de la cérémonie (nommée Gurirr Gurirr) furent transmis en quatre langues: Kija, Worla, Ngarinyin et Mirriwoong selon les territoires traversés par l'esprit, ce qui rendit le rituel difficilement compréhensible pour Thomas. N'étant pas Kija, Thomas ne possédait pas non plus l'autorité rituelle pour transcrire les images sous forme de peintures et mettre en place la cérémonie. Il dut contacter un vieil initié de Warmun, Paddy Jaminji, originaire de Bedford Downs, connu dans la communauté pour ses talents de peintre sur différents supports (boomerang, coolamon, objets divers) qu'il vendait aux propriétaires des boutiques de la région (Macha, 1997). Une collaboration se mit alors en place entre Thomas et Jaminji pour retranscrire, sous forme de 
peintures sur panneaux, les images issues du rêve de Thomas. Les premiers panneaux peints proviennent donc d'un travail coopératif dans lequel Thomas guidait la main de Jaminji. Quelques années plus tard, Jaminji commença à peindre des œuvres sur toile qui ne se référaient pas à la cérémonie Gurirr Gurirr, mais à d'autres sites de la région. Il fut suivi dans cette démarche par Thomas avec un succès considérable ${ }^{3}$. Leur influence est palpable chez tous les autres peintres de la région (Ryan et Akermann, 1993). À partir du milieu des années 1980, les peintures se sont partiellement détachées de leur cadre rituel et une petite production d'œuvres sur toile, collectionnées par les musées et les galeries d'art, se mit en place.

6 Si le mouvement artistique du Kimberley Oriental est étroitement affilié à la peinture du désert à travers la figure de Rover Thomas, il s'en démarque toutefois sur plusieurs points-clés. Formellement, les peintures contemporaines de Warmun sont composées de larges champs de pigments naturels (et non acryliques), séparés par de grandes lignes de pointillés blancs qui partagent la toile. Ce qui frappe au premier abord est l'absence de motifs clairement identifiables, les lignes mises à part. Ces œuvres apparaissent comme une version épurée de l'art du désert: un fond sans motifs iconographiques facilement reconnaissables, le fond n'est pas constitué de points, mais d'une texture d'ocre, mate, brillante ou translucide. Il s'agit en fait toujours d'une vision aérienne d'un paysage, mais ici la trace n'est pas toujours visible, elle se confond parfois avec le sol lui-même et sa texture, la peinture elle-même devient donc trace. Plutôt que de figurer des itinéraires entre différents points, les peintures montrent plus souvent un lieu unique, où s'est incarné un ancêtre. Elles font appel à une mémoire ancienne, celle de la formation des paysages.

$7 \quad$ Le travail sur les mélanges de pigments permet de créer une densité et une saturation des couleurs dans les larges aplats du tableau, mats par endroits, et translucides à d'autres, donnant l'impression que l'œuvre ne montre pas seulement la surface du paysage, mais le traverse pour accéder à ce qu'elle cache, des êtres mythiques qui y reposent après leurs actions ordonnatrices. Les formes qui apparaissent sur la toile sont inspirées par les configurations du paysage lui-même, mais les distances et les proportions ne sont pas respectées. Il s'agit d'une interprétation libre qui donne à voir les transformations et les tensions existant dans un espace créé par des actions surnaturelles (actes mythiques à l'origine des lieux). Dans le Kimberley, le paysage est pris comme un tout, un concept holistique, ce n'est pas un ensemble de traces, mais comme une trace elle-même de l'activité ancestrale. Les peintures du Kimberley, depuis Rover Thomas, comprennent peu ou pas de signes, mais sont marquées par un rapport topologique au lieu (et non pas topographique), c'est-à-dire la définition d'un espace par ses propriétés, ses déformations et ses transformations plus que par la description et la mesure de ses formes visibles (Glowczewski et Guattari, 1986).

Pour reprendre la tripartition des signes de Peirce (1955), les peintures n'entretiennent pas de relations de type iconique (basées sur une ressemblance formelle avec le référent) ni de type symbolique (basées sur une correspondance conventionnelle). En revanche la relation "indicielle» au référent rencontre ici un écho favorable. Peirce définit l'indice comme un élément visuel entretenant un rapport de cause à effet avec l'idée ou l'objet auquel il renvoie, par exemple le rapport de la fumée au feu (Peirce, 1955 ; Dubinskas and Traweek, 1984). On se trouve donc ici à mi-chemin de la présence et de la représentation. Les peintures de Paddy Bedford ou Rover Thomas donnent à voir des paysages. Elles portent en elles-mêmes à la fois la substance de ces paysages 
(les ocres proviennent du territoire dépeint) et la trace de la main de l'artiste (comme résidu du geste et de la performance picturale), et deviennent "affectées » dès lors qu'elles sont nommées par l'artiste matérialisant la relation existentielle qui existe entre le peintre et le site peint. Cette relation se présente comme une contraction de la mémoire de l'artiste et de celle du lieu, qui fusionnent dans un rapport de contiguïté qui rattache un peintre à sa terre, les deux entités (personne et environnement) partageant la même essence. L'utilité heuristique de l'indice permet de dépasser l'opposition entre présence et représentation, et établit un rapport entre les éléments visuels de la peinture et l'action génératrice conjointe du peintre (la performance) et des éléments mythiques qui en sont à l'origine.

Figure 1Rover Thomas, Yari Country - 1989.

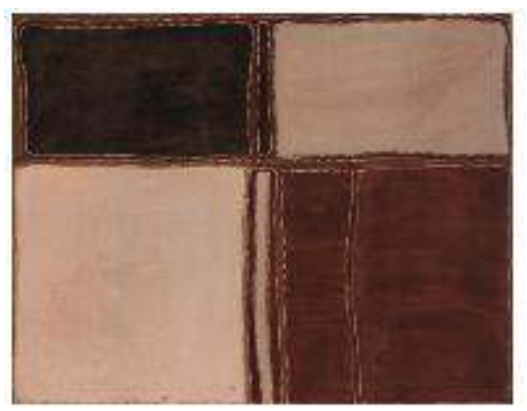

Pigments naturels sur toile $160 \times 200 \mathrm{~cm}$

(COLLECTION NATIONAL GALLERY OF VICTORIA)

Graphique 1 : Yari Country 1989

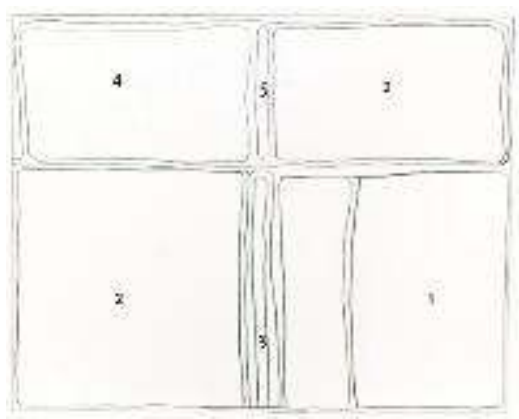

1- Wala (désert)

2- Yari (billabong empoisonné)

3- Club du vieil homme

4- Terres brûlées

5- Promontoire où le vieil homme s'est abreuvé

(๔ Arnaud Morvan) 
Figure 2Paddy Bedford, Mc Phee Hole - 1998.

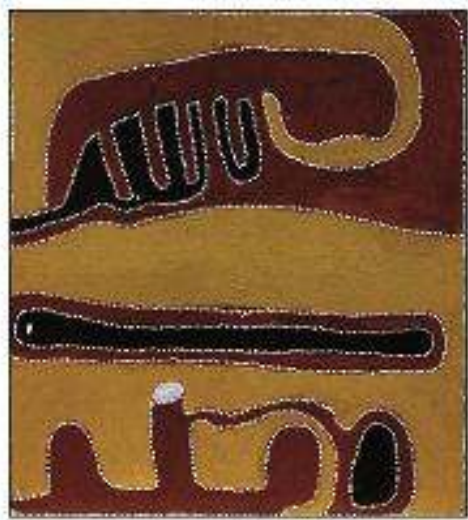

Pigments naturels sur toile $135 \times 122 \mathrm{~cm}$ (Collection privée)

Graphique 2 : Mc Phee Hole 1998

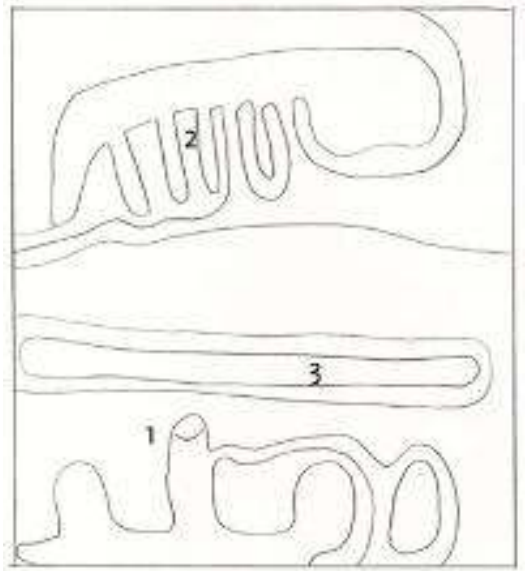

1- NgAYILANJI

2- GROUPE D'HOMMES PÉTRIfIÉS

3- RELIEF SÉPARANT NGAYILANJI DU gROUPE D'HOMMES

(C Arnaud Morvan)

Figure 3Paddy Bedford, Cockatoo - 1999.

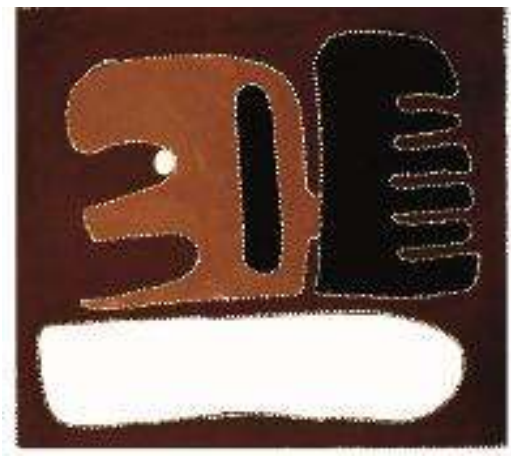

Pigments naturels sur toile $135 \times 122 \mathrm{~cm}$ (Collection privée) 
Graphique 3 : Cockatoo 1999

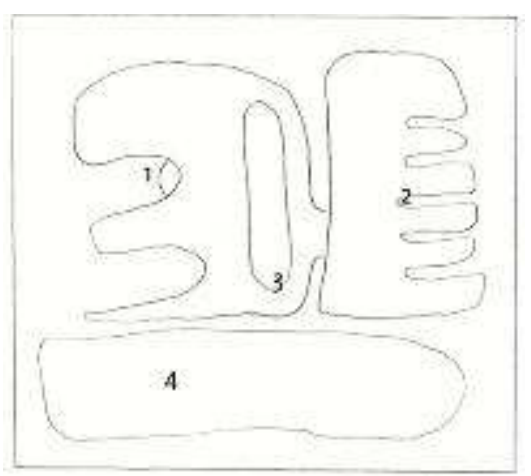

1- Ngayilanji

2- Groupe d'hommes pétrifiés

3- Relief séparant Ngayilanji du groupe d'hommes

(๔) ARNAUD MORVAN)

Figure 4

Paddy Bedford, Jawoorraban / Cockatoo Dreaming - 2000.

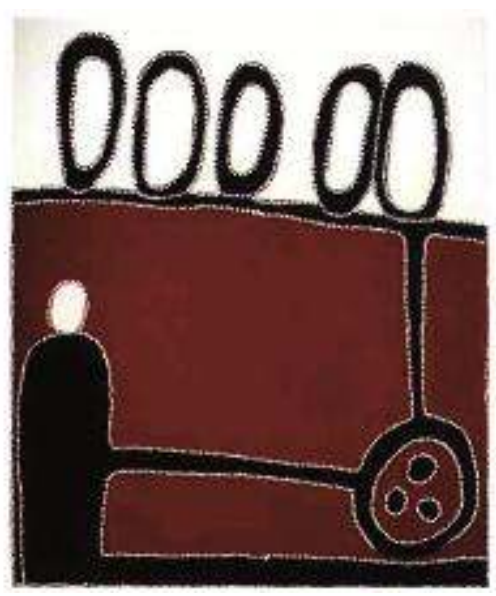

Pigments naturels sur toile $135 \times 122 \mathrm{~cm}$ (Collection privée) 
Graphique 4 : Jawoorraban / Cockatoo Dreaming 2000

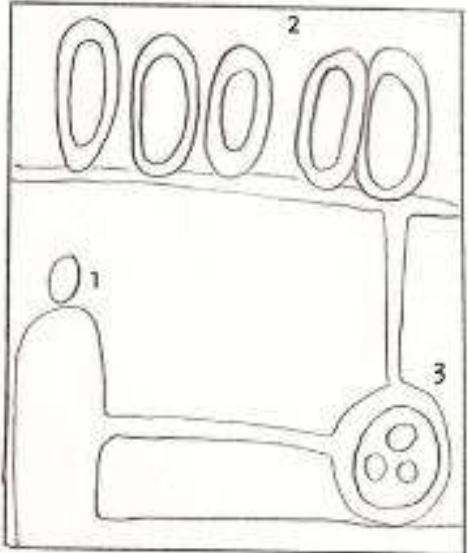

1- Ngayilanji

2- Groupe d'hommes pétrifiés

3- Lieu de la rencontre entre Ngayilanji et le groupe d'hommes

(৫) ARNAUD MORVAN)

Figure 5

Paddy Bedford, Jawoorraban / Cockatoo Dreaming - 2000

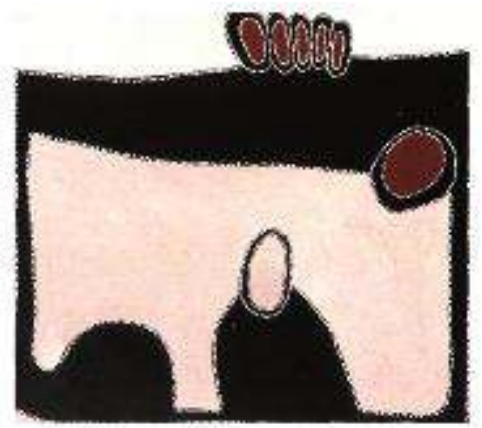

Pigments naturels sur toile $180 \times 150 \mathrm{~cm}$ (Collection privée)

Graphique 5 : Jawoorraban / Cockatoo Dreaming 2000

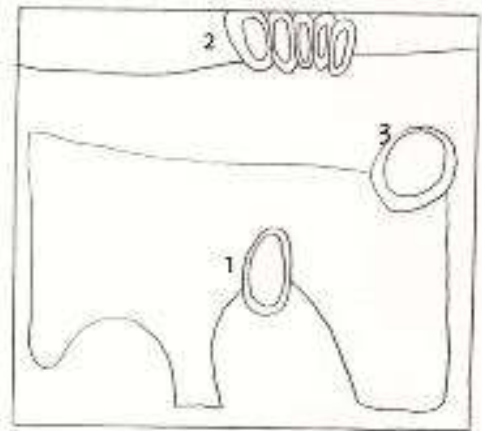

1- Ngayilanji

2- Groupe d'hommes pétrifiés

3- Lieu de la rencontre entre Ngayilanji et le groupe d'hommes

(c) Arnaud Morvan) 

par Paddy Bedford ${ }^{4}$ entre 1998 et 2002, donnent à voir une série de variations dans la représentation de l'espace lié au site de Jawoorraban, de corniches rocheuses dentelées sur les contreforts des Durack Ranges qui montrent la pétrification d'un groupe d'ancêtres et leur fusion dans le paysage. D'après Paddy Bedford, le site est issu de la rencontre entre l'ancêtre totémique Ngayilanji (Cacatoès blanc), venant de l'est, et un groupe d'hommes marchant en direction du sud. Ngayilanji les empêcha de continuer leur route vers les terres des Gooniyandi, au sud-ouest du territoire kija. Ils s'arrêtèrent à Jawoorraban et Ngayilanji leur bloqua le passage. Il escalada ensuite une colline et entonna un chant joonba qui changea les hommes en pierre à Jawoorraban (Kofod, 2006, 84).

10 Les peintures de Bedford montrent la pétrification des hommes et leur fusion dans le paysage. Les tableaux décrivent le site de Jawoorraban et la région environnante en vue aérienne. Plusieurs éléments saillants reviennent de manière récurrente dans ces peintures intitulées Mc Phee Hole, Jawoorraban ou Cockatoo Dreaming. On peut identifier au moins trois à quatre motifs récurrents: un cercle blanc plein représentant le personnage de Ngayilanji, une forme dentelée ou une série d'ovales représentant les hommes pétrifiés formant une crête sur la montagne, une ligne noire qui sépare les deux figures, un cercle rouge qui montre le groupe d'hommes marchant vers le sud.

11 Le tableau Mc Phee Hole 1998 reprend de manière assez précise la topographie des lieux (chose assez rare chez Paddy Bedford) en instaurant un code de trois couleurs (jaune, rouge et noir) qui définissent la hauteur du relief du plus bas au plus élevé : la plaine en jaune, la colline en rouge et la montagne en noir. On y voit Ngayilanji se dresser sur une colline face au groupe d'hommes marchant vers le sud qui apparaissent pétrifiés dans les crêtes des Durack Ranges. Les gorges qui serpentent au pied des montagnes et entre les collines sont représentées avec précision pour former un paysage ciselé. La peinture Cockatoo 1999, réalisée l'année suivante, présente la même scène, mais selon un angle différent comme si la toile avait effectué une rotation vers la droite. Les trois éléments nommés précédemment demeurent présents, mais le relief y apparaît plus schématisé, d'une expression plus brute des paysages. Seuls les traits les plus saillants sont désormais visibles : le cercle blanc, les formes dentelées, séparées par la ligne noire. Les références topographiques se sont atténuées pour céder la place à ce qui ressemble davantage à une image mentale du lieu. Cette impression se renforce dans la série suivante qui date de l'année 2000. La forme dentelée est remplacée par une série de cinq ovales rouges qui font face à la forme blanche de Ngayilanji. Un cercle rouge apparaît entre les deux figures et indique l'emplacement où les personnages se sont rencontrés avant d'être changés en pierre. Cette séquence devient encore plus apparente dans la peinture suivante où le cercle en question est lui-même rempli de plusieurs cercles noirs et se trouve être le point de départ de deux lignes qui le relient aux deux autres figures du tableau (les ovales des hommes pétrifiés et la figure de Ngayilanji).

Dans les peintures de Paddy Bedford et dans certaines toiles de Rover Thomas, les motifs peuvent se lire simultanément comme un plan ou comme une élévation, comme une transformation optique dans l'espace de la toile. L'espace de la peinture effectue des rotations autour de l'axe de l'horizon, les formes semi-abstraites des paysages accidentés du Kimberley se lisent alternativement à plat ou de profil. Une ligne d'horizon peut ainsi se changer en ligne de démarcation entre une plaine et un 
escarpement rocheux, un peu comme si l'œil effectuait des rotations autour de l'objet. Ce procédé donne à voir la nature mouvante d'un paysage qui n'a pas la platitude du désert, mais joue sur les contrastes, la partition du ciel et de la terre, les zones d'ombre et de lumière, gorges et falaises, terrains ouverts ou abrités. Dans les représentations des hommes pétrifiés du site de Jawoorraban, les formes ovales proéminentes évoquent simultanément des rochers arrondis (boulder) dressés, mais qui peuvent aussi être vus à plat, comme de longues pierres plates. Certaines peintures font preuve d'un sens $d u$ déplacement vertigineux qui reflète une fois de plus la plasticité d'un paysage en formation, l'énergie instable des forces qui l'animent.

\section{La trace comme mémoire du lieu}

Les peintures ont été définies comme des représentations de type indiciaire, c'est-àdire qu'elles partagent quelque chose avec leurs référents. Cette relation est de nature métonymique, la peinture est un fragment ou une répercussion d'une action ancestrale plus vaste liée à la création des paysages. La nature indiciaire de la représentation suppose une induction, un procédé qui implique de remonter d'une manifestation de quelque chose à ce qui en est la source. Ce procédé tourne autour du concept de «trace » qui constitue la principale convention de figuration dans les régions du désert et, depuis Rover Thomas, dans le Kimberley.

Dans une série de recherches sur les arts de la mémoire et la biologie des images, l'anthropologue Carlo Severi présente une méthode pour analyser la relation entre certains types d'images et certains procédés de mémorisation (notamment la mémorisation des noms - toponymes et anthroponymes). Severi $(2003,121)$ propose pour cela de croiser l'établissement de séquences ordonnées et la mise en place de saillance visuelle à l'intérieur d'une image selon une «relation mnémonique». Il explique que le rôle propre à l'image dans cette perspective implique «un processus d'intensification (cognitive et mnémonique) de la représentation visuelle par la mobilisation de ses parties invisibles ».

La référence à l'intensification visuelle par la mobilisation des parties invisibles d'une image rencontre un fort écho dans la conception kija de la trace (gamerre) et se révèle particulièrement pertinente pour comprendre les composantes mnémoniques de l'art du Kimberley oriental. Quels sont les actes de regard à l'œuvre dans les peintures et quel est le lien entre cette opération et la mémoire ? L'exercice mnémonique semble consister ici à relier la définition visuelle de la trace - comme suite d'empreintes ou de marques que laisse le passage d'un être ou d'un objet - à sa définition temporelle (ce qui subsiste d'une chose passée). La trace donne à voir ce qui n'est plus là et oblige l'œil de l'observateur à reconstituer l'action créatrice en se remémorant les éléments manquants ou invisibles. Le travail de mémoire se fait par la recherche de la chose absente, pour restituer son parcours. Cette opération s'accomplit à la fois chez le performer (qui lui-même peint à partir d'un souvenir, d'une révélation, ou de la mobilisation de sa propre mémoire) et chez le spectateur qui doit recréer l'image à partir de la trace dans un travail mémoriel. Ces éléments manquants s'accompagnent, outre une saillance visuelle, d'éléments narratifs indexicaux de trois types: le titre de l'œuvre (généralement un toponyme ou un anthroponyme), le récit associé aux lieux peints, les chants rituels liés à certains sites. Ces trois éléments peuvent être présents 
ensemble ou séparément pour une peinture donnée, ils s'appliquent aussi bien aux peintures rituelles sur panneaux qu'aux œuvres contemporaines sur toile.

La peinture de Paddy Bedford, intitulée Emu Dreaming 2003, montre l'association d'une image et d'un chant qui s'applique ici non pas seulement à une mémoire mythique, mais à l'enregistrement d'un événement historique.

Figure 6Paddy Bedford, Emu Dreaming - 2003

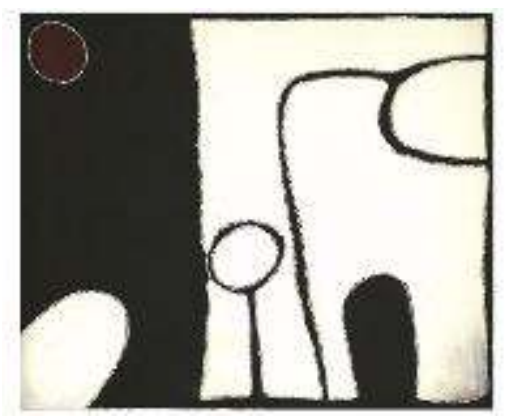

Pigments naturels sur toile, $150 \times 180 \mathrm{~cm}$

(COLLECTION NATIONAL GALLERY OF AUSTRALIA)

Graphique 6 : Emu Dreaming 2003
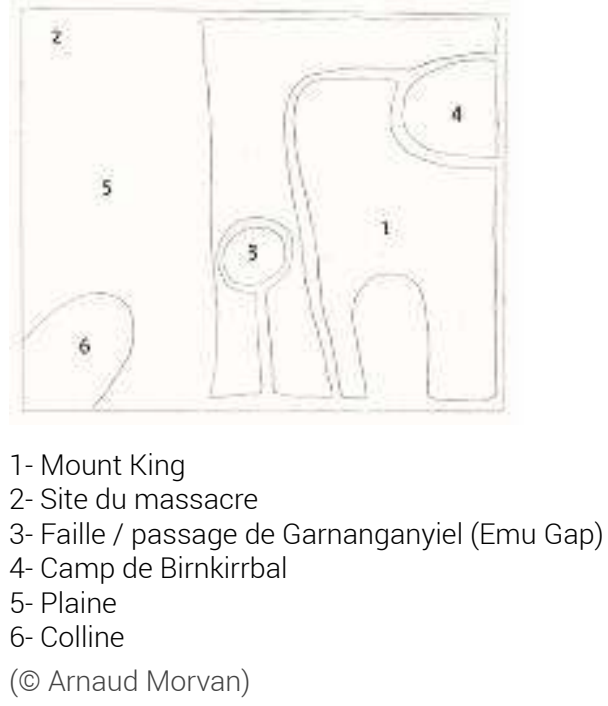

Bedford a peint le site du rêve Emeu à Mount King dans une vingtaine de toiles sur une durée de huit ans entre 1998 et 2005. Gardien rituel du site, il possède une connaissance approfondie des événements ayant façonné l'endroit. Le tableau intitulé Emu Dreaming 2003 conservé à la National Gallery of Australia se réfère au mythe de l'Emeu qui fonde l'alternance du jour et de la nuit, des moments d'action et de repos. L'histoire raconte comment deux ancêtres, Garnanganyel (Emeu) et Birnkirrbal (Dinde), voyageaient vers l'est et furent stoppés par une chaîne de montagnes à Mount King. Les deux ancêtres s'engagèrent dans un corridor qui s'enfonçait dans la montagne. Le soleil se couchant, Birnkirrbal décida d'établir un campement pour passer la nuit. Garnanganyel choisit de continuer malgré la nuit, se perdit et resta bloqué à jamais dans la montagne. L'endroit où Garnanganyel trouva la mort est aujourd'hui marqué par une grande faille qui entaille le flanc de Mount King. 
Le tableau montre une vue large du paysage de Mount King qui apparaît en blanc sur la droite de la peinture, la plaine en contrebas est peinte en noir. Les différents sentiers et campements créés par les deux ancêtres totémiques y apparaissent comme des intrusions noires dans la roche (blanche) de la montagne. On remarque aussi une tache rouge, un cercle excentré dans la partie haute de la toile. Ce cercle rouge qui apparaît dans certains tableaux de Bedford depuis 1998 indique l'emplacement d'un lieu nommé Goordbelayin («l'endroit où beaucoup furent tués »), au pied de la montagne. Il s'agit de l'endroit où plusieurs membres de la famille de Paddy Bedford ont trouvé la mort, empoisonnés à la strychnine puis brûlés par des fermiers blancs, quelques années avant la naissance de l'artiste (au début des années 1920). L'histoire de ce massacre, comme des dizaines d'autres qui ont été perpétrés dans la région, fut cachée aux Blancs jusqu'à une période très récente. Le récit de la tuerie et du voyage des esprits des morts fut transmis sous forme de chants et de danses dans tous les campements aborigènes de la région, généralement installés autour des fermes d'élevage dans les années 1930 et $1940^{5}$. Ce n'est qu'en 2000, après avoir réalisé plusieurs peintures du site de Bedford Downs, que Paddy Bedford décida de rendre publics les chants partiellement oubliés associés à l'endroit. Le passage reproduit ici se réfère au moment où les esprits quittent leur corps, gravissent les flancs de Mount King et se retournent dans le soleil couchant pour regarder leur propre cadavre brûler. La suite du chant évoque le voyage des esprits vers l'ouest à la recherche d'un chaman capable d'entendre le récit de leur mort tragique.

'Ngindil ba 'ngindil ma'nay, 'Ngindil ba 'ngindil ma'nay,

arrirri ma'naay, dilirri ma'naay, marrirri ma'naa dilirri ma'naa

Ngindil ba ngindil manaa dilirri ma naa dilirri ma'naa.

- L'étincelle, l'étincelle brille; l'étincelle, l'étincelle brille

- La brillance du soleil couchant, le rougeoiement du bûcher, la brillance du soleil

couchant, le rougeoiement du bûcher

- L'étincelle, le rougeoiement du bûcher, le rougeoiement du bûcher.

Cette variation chantée autour de la lumière rouge se matérialise dans la peinture sous la forme d'un cercle d'ocre rouge surligné de points blancs dans la partie supérieure gauche de la peinture. La composition du tableau semble conçue pour attirer le regard vers ce cercle rouge, unique élément de couleur dans la toile, au cœur d'un paysage noir et blanc. Ce cercle agit également comme un contrepoint par rapport aux différentes traces laissées par les êtres totémiques dans la montagne, en particulier le site de l'Emeu, cercle blanc, qui donne son nom à la peinture. L'œuvre semble construite de telle sorte que l'œil de l'observateur fasse des aller-retour entre les formes blanches (montagne, éléments mythiques) et le cercle rouge en contrepoint (massacre, élément historique) comme pour mentionner une présence dissonante dans ce paysage mythique.

On constate ici que le chant évoque un événement sous la forme non pas d'une narration, mais d'une image. La deuxième ligne de chant établit une relation entre la brillance du bûcher et celle du soleil couchant. Elle indique par là même la position des esprits (auteurs du chant), situés entre ces deux points et décrit un mouvement allant du site du massacre et en direction du soleil couchant à l'ouest, c'est-à-dire entre l'endroit de la mort et celui de la destination finale des esprits, à l'ouest où se situe une île des morts dans les cosmologies du Kimberley. Le chant permet de percevoir ce qui est a priori invisible à l'œil, le mouvement des esprits vers le soleil, en direction de l'ouest. 
20 Le massacre n'est donc pas évoqué comme un site figé, mais plutôt comme une mise en scène d'une mémoire en mouvement, symbolisé par le déplacement des esprits qui s'échappent du lieu de massacre pour transmettre l'histoire de leur mort. Nous constatons ici que le chant participe à l'intensification d'une saillance visuelle (correspondance rouge chantée et rouge peint), puis contribue à la mise en mouvement de l'image en suggérant un acte de regard qui prend le site du massacre comme un point de départ. Cette mise en mouvement de l'image par le chant participe à la construction d'une mémoire. Pour vérifier cette hypothèse, il nous faut nous intéresser à l'interaction des peintures et des chants dans l'acte rituel dont elles sont issues.

\section{Le chant rituel comme élément indexical}

21 Comme nous l'avons vu, les peintures sur toile sont directement issues des peintures sur bois, utilisées comme objet rituel dans des cérémonies publiques de type joonba. Ces cycles de chants et de danse sont à l'origine des peintures contemporaines et constituent l'un des éléments les plus flexibles du complexe mythico-rituel australien. Les chants sont souvent révélés à un individu pendant son sommeil par l'esprit d'une personne décédée, un juwari. Le contenu d'un joonba est très souple. Il a pour protagonistes trois types de personnages: être mythique, esprit, personnages historiques ou contemporains évoqués sous la forme d'une suite de toponymes, d'anthroponymes, de verbes et d'indications de déplacement et de direction, le tout composant généralement un itinéraire entre différents sites qui ne se limitent pas aux territoires claniques du dépositaire $d u$ chant $^{6}$. Les chants se présentent comme la remémoration d'événements plus ou moins récents, dont certains auraient été oubliés. Les joonba entretiennent de ce fait des relations étroites avec le passé d'une part et avec le contexte social et politique de leur révélation d'autre part.

Pour illustrer notre propos, comparons par exemple le chant du joonba «Binyjirmin Nawan ", enregistré dans la communauté de Bow River en juillet 2007, et le panneau peint qui l'accompagne. Cette cérémonie à portée autobiographique retrace le parcours du groupe d'artistes nommé Jirrawun dont Paddy Bedford était le leader depuis sa création en 1998. Ce joonba, créé entre mars et juillet 2007, est composé de quatre chants qui évoquent différents lieux et événements liés à la création de ce groupe et aux entités mythiques qui y sont associées ${ }^{7}$.

23 Les cérémonies se tiennent de nuit ou au coucher du soleil sur un terrain cérémoniel constitué d'un espace de danse, qui forme un rectangle d'environ $5 \mathrm{~m} \times 8 \mathrm{~m}$, équipé à l'une de ses extrémités d'un écran en feuillage, d'où sortent les danseurs au début de chaque séquence. Les participants sont scindés en deux groupes positionnés de part et d'autre du terrain de danse : le chanteur principal et son groupe sont situés à l'avant de la scène, les danseurs se tiennent à l'opposé derrière l'écran. C'est le chant qui déclenche et commande la danse et non l'inverse. Les danseurs, qui portent sur leurs épaules des images peintes sur panneau, n'apparaissent qu'une fois le chant entonné. Leurs mouvements constituent une extension visuelle du chant. Ils suivent ensuite le rythme des bâtons de percussion, en se rapprochant en ligne du groupe de chanteurs devant lequel ils s'immobilisent avant d'effectuer le même trajet en sens inverse. Les mouvements sont les suivants : frapper le sol du pied en avançant ou en reculant avec des périodes d'arrêt et un rapprochement ou un éloignement avec les autres danseurs. Alors que les mouvements avant-arrière sont commandés par les chants, les battements 
des jambes sont parfaitement synchronisés sur le son des bâtons, les clap-sticks, grâce auxquels les chanteurs dirigent et contrôlent le corps des danseurs. Le chant, la musique et les déplacements des danseurs sont rythmés par l'alternance de périodes d'accélération, de ralentissement, d'arrêt, de redémarrage. Chaque percussion des bâtons correspond à un pas et chaque pas correspond à une percussion. L'ensemble est accompagné par le bruit sourd des pieds qui martèlent le sol et soulèvent un épais nuage de poussière, masquant peu à peu les jambes des danseurs jusqu'à les faire parfois entièrement disparaître ${ }^{8}$.

Le panneau est maintenu sur les épaules du danseur de telle manière que sa tête apparaisse au bas du panneau comme "encadrée ». La peinture Lumugul reprend la forme de la colline sur laquelle se dresse le rocher Lumugul, incarnation du lézard à langue bleue. Le panneau est peint d'un large demi-cercle à l'ocre jaune entouré de noir, et surplombé par un rectangle jaune sur lequel se détache la silhouette noire d'un lézard à la queue courte et la tête volumineuse. Tous les éléments à l'exception du lézard sont rehaussés de points blancs. Le panneau montre donc à la fois un rocher et un ancêtre dans une représentation semi-figurative qui se déplace avec les danseurs. Il suit les mouvements du corps et se déplace donc légèrement de gauche à droite et de haut en bas. Dans le cas de la danse Lumugul, le danseur s'immobilise plusieurs fois et tourne lentement sa tête vers l'arrière. La performance dure généralement deux ou trois minutes jusqu'à la fin du couplet qui se termine par le retour du danseur derrière l'écran de feuillage. Après une courte pause d'une minute ou deux, l'opération se renouvelle avec le retour d'un danseur équipé d'un nouveau panneau. Précisons que certaines séquences ne comprennent pas de panneaux et sont seulement dansées. La performance se présente donc comme une mise en séquences d'images mouvantes sur panneaux, qui correspondent à des noms de lieux chantés et des personnages représentés de manière figurative (sous la forme d'une silhouette ou d'un visage) ou abstraite (représentation mentale de certains traits d'un paysage).

Le chant Lumugul a été retranscrit de la manière suivante :

Ligne A : Lumuguliyi, lumuguliyi, wad-garri nyanininya.

- The blue tongue, the blue tongue, when she was looking back (i.e. when she was a

woman in dreamtime).

- Le lézard à langue bleue, le lézard à langue bleue, quand il regardait en arrière.

Ligne B : Wad-garri nyanininya, Ruguniyi, Rugun.

- When she was looking back, at Crocodile Hole, at Crocodile Hole9.

- Quand il regardait en arrière, vers Rugun (antre du crocodile).

L'ordre d'apparition des mots dans une ligne de texte et la situation des chants les uns par rapport aux autres jouent un rôle important, car il permet de définir une suite de lieux, un itinéraire qui contient souvent une partie importante du sens du rituel (Morvan, 2010). Le nombre de chants dépend généralement de la longueur du trajet effectué par les personnages dans le joonba et peut comprendre entre trois et quarante chants constitués généralement de deux lignes de texte. Comme l'ont souligné de nombreux linguistes, les chants aborigènes sont extrêmement difficiles à transcrire. Les mots sont déformés et étirés en fonction de la ligne mélodique, certains mots n'ont pas de sens connu, d'autres appartiennent à des langues de groupes voisins, parfois non parlées par les compositeurs du chant. Le compositeur d'un chant joonba adapte le texte à la mélodie et non l'inverse. Le rythme frappé par les clap-sticks est assez rapide et oscille entre quatre-vingt-dix et cent dix battements par minute. La ligne mélodique est constituée d'une série de descentes tonales longues et courtes selon un schéma dans lequel une descente courte s'intercale entre deux descentes longues. Les structures 
mélodiques et textuelles des joonba kija semblent correspondre à celles des joonba de leurs voisins ngarinyin dont les chants ont été étudiés par la linguiste et ethnomusicologue Sally Treloyn. Les chants sont constitués généralement de deux lignes de texte (texte-ligne $\mathrm{A}$ et texte-ligne $\mathrm{B}$ ) qui sont répétés cycliquement tout au long d'une séquence sous la forme AABBAABBAABB. À partir de cette structure symétrique Treloyn explique que les combinaisons d'éléments textuels et mélodiques sont agencées de manière à introduire des irrégularités dans le parallélisme des deux lignes A et B, puis entre le texte et la structure mélodique (Treloyn, 2003). Ce procédé consiste à créer un déséquilibre dynamique, un mouvement de flux et de reflux entre les différentes lignes-textes, juxtaposées puis entrecroisées. Les chants semblent participer à ce jeu de mise en présence, de juxtaposition, de montage, et d'associations libres entre les multiples niveaux de sens et les différents types de temporalité, à commencer par le temps du mythe et celui des acteurs contemporains.

Dans le chant Lumugul, le sujet figure de manière proéminente au début de la ligne de texte, son occurrence est doublée, il conditionne généralement le reste du chant. Ici le sujet, premier mot de la ligne A 'Lumuguliyi', est une déformation du nom 'Lumugul', qui désigne un être mythique (femme lézard à langue bleue) associé à une proéminence rocheuse dans la plaine de Lissadell à l'ouest de Bow River : l'endroit (Naminuwarlin) où s'est pétrifié l'ancêtre Lumugul marque la frontière entre les territoires des groupes de langue kija et mirriwung. Lumugul est clairement associé au langage et à la narration ; c'est une figure importante dans la cosmologie kija qui fait figure de matrice narrative. Peggy Patrick, l'une des gardiennes du site Neminuwarlin et conceptrice du chant, en explique une partie du sens :

Lumugul est notre ancêtre du Rêve, c'est Neminuwarlin (nom de l'endroit où Lumugul s'est matérialisé). Ce Lumugul nous a donné des histoires... (des choses) pour produire des histoires, comme l'histoire de Binyjirmin et le reste. C'est parce qu'il est originaire de Crocodile Hole-Rugun (nom d'un trou d'eau et d'une communauté aborigène). Il possède l'histoire pour Crocodile Hole, pour Rugun. Il nous a donné une histoire pour Rugun à travers Naminuwarlin. Ce Naminuwarlin, nous l'appelons Lumugul. Le mot « Wad » (chant ligne A et B) signifie « regarder en arrière », il regarde en arrière vers cet endroit Rugun. C'est l'histoire pour ce joonba (...) il regarde en arrière vers nous à nouveau ${ }^{10}$.

La ligne B du chant se termine par le mot Rugun. L'agencement des deux lignes de textes $A$ et $B$ suggère une relation entre le personnage de Lumugul et le lieu Rugun où ont été créées les premières peintures. Les mots wad-garri nyanininya (« regarder en arrière vers nous») terminent la ligne-texte $\mathrm{A}$ et amorcent la ligne-texte $\mathrm{B}$. Cette répétition indique la centralité de l'action de se retourner pour regarder en arrière, une figure poétique liée à l'acte narratif et au passé dans les cultures semi-nomades du nord-ouest australien. Le chant étudié établit donc un parallèle entre l'anthroponyme Lumugul et le toponyme Rugun, entre un ancêtre du Dreaming (représentant une matrice narrative) et le groupe d'artistes installé à Rugun ${ }^{11}$, entre le niveau de narration mythique et le niveau historique. Une correspondance s'instaure entre d'une part l'ancêtre Lumugul qui se retourne et, d'autre part, les peintres (ligne B) qui réinterprètent leur propre passé à la fois dans ce joonba et dans leur démarche artistique (à travers des références à la période coloniale - massacres-dans les peintures). Ainsi, le chant associé aux peintures tend à indexer non seulement des lieux et des noms, mais aussi une relation entre deux niveaux de temporalité mythique et historique. Le lien entre images et chants dans le joonba permet de relier des lieux et des événements distants dans le temps et l'espace. L'image devient alors un support 
visuel pour connecter plusieurs événements liés à un même lieu. Cette relation entre image et événement par le chant ne s'élabore pas sur un modèle linéaire ou unidirectionnel, mais plutôt, comme les variations de lignes mélodiques du joonba, de manière instable et anachronique.

En tant qu'images mentales de lieux, les peintures kija sont composées à la fois d'éléments visuels et verbaux qui participent à l'élaboration d'une mémoire. Si la peinture apparaît comme une condensation de l'espace sous forme schématique, le chant semble être une version synthétique d'un récit. Images peintes ou chantées s'inscrivent dans un même processus mnémonique basé sur une représentation indiciaire d'un lieu. Le chant participe ainsi à l'intensification visuelle d'une forme en tant qu'élément indexical, c'est-à-dire qu'une partie de la signification d'un lieu dépend de son association à un chant. Les chants participent à une mise en mouvement des images à plusieurs niveaux. D'abord de manière concrète lorsque les danseurs portent les peintures sur leurs épaules pour donner à voir le chant: dans le cas du panneau Lumugul, la peinture bouge d'avant en arrière et se retourne littéralement avec le danseur. Ensuite le chant met en scène, dans sa structure même, un déplacement basé sur l'introduction d'irrégularités et de déséquilibres dans la ligne mélodique et dans la relation entre des personnages et des lieux. Ce principe de mouvement se transpose dans les peintures sur toile par différents procédés visuels : les différences de texture d'ocres qui créaient de la profondeur dans l'espace de la toile en alternant transparence et opacité, la perspective dynamique qui alterne les vus aériennes et latérales d'un même objet, enfin la composition des œuvres qui créer des mouvements de regard entre les éléments mythiques ou historiques.

La relation entre les chants et les œuvres peintes permet de repenser la peinture non pas comme l'image figée d'un lieu, mais plutôt d'un déplacement, d'un mouvement, d'un flux temporel. Les performances et les chants actualisent plusieurs niveaux mémoriels mythiques, historiques, biographiques à l'intérieur des œuvres donnant à voir une temporalité instable, dynamique, faite de flux et de reflux qui semble alors « creuser » le temps.

\section{BIBLIOGRAPHIE}

BARWICK, L., 1989, Creative (ir)regularities: The intermeshing of text and melody in performance of Central Australian song, Australian Aboriginal Studies, 1: 12-28.

DEsColA, Philippe, 2005, Par delà nature et culture, Paris, Gallimard.

Descola, Philippe, 2010, Introduction. Manière de voir, manière de figurer, in Descola (dir.), La Fabrique des images. Visions du monde et formes de la représentation, Paris, Somogy éditions/MqB, p. 11-18.

DoLK, M., 2006, Are we strangers in this place?, in R. Storer (ed.), Paddy Bedford, Sydney, Museum of Contemporary Art Press: 17-49. 
DUBINSKAS, F.A. \& TRAWEEK, S., 1984, Closer to the ground: a reinterpretation of Walbiri iconography, Man, 19: 15-30.

DUSSART, F., 2000, The politics of ritual in an Aboriginal settlement. Kinship, gender, and the currency of Knowledge, Washington, Smithsonian Institution.

DusSART, F., 2010, De la Terre à la toile : peintures acryliques de l'Australie centrale, in Philippe Descola (dir.), La Fabrique des images. Visions du monde et formes de la représentation, Paris, Somogy éditions/MqB, p. 139-145.

GLOWCZEWSKI, Barbara, 1996, Histoire et Ontologie en Australie aborigène, L'Homme, 137, p. 211-225.

GLowCZEWSKI, Barbara, 1991a, Du rêve à la loi chez les Aborigènes. Mythes, rites et organisation sociale en Australie, Paris, Presses Universitaires de France.

GLowCZEWSKI, Barbara, 1991b, Yapa : Peintres aborigènes de Balgo et Lajamanu, Paris, Baudoin Lebon. GLOWCZEWSKI, Barbara et GUATTARI, Félix, 1986, Les Warlpiri, discussions avec Félix Guattari (1981-1983), Chimères, 1, p. 4-37.

KofoD, Frances, 2006, Paddy Bedford's stories, in R. Storer (ed.), Paddy Bedford, Sydney, Museum of Contemporary Art Press: 72-98.

LANGTON, M., 2002, Hungry ghost: Landscape and memory, in Blood on the Spinifex, exhibition catalogue, Melbourne, The Ian Potter Museum of Art, The University of Melbourne: 12-15.

Lommel, A., 1997, The Unambal, A tribe in NorthWest Australia, [trad. Ian Campbell] Carnarvon Gorge, Takarakka Nowan Kas Publication, [1st publ., 1952].

MACHA, M., 1997, Paddy Jaminji, l'Artiste, in S. Crossman et J.-P. Barou (éds), Peintres aborigènes d'Australie, catalogue de l'exposition, Montpellier, Indigène Éditions, p. 104-6.

MoRvan, Arnaud, 2007, Retours d'exotismes. Présence des artistes aborigènes australiens en France, in Barbara Glowczewski et R. Henry (éds), Défi indigène. Entre spectacle et politique, Paris, Aux Lieux d'être, p. 125-149.

MoRVAN, Arnaud, 2009, Performing Landscape and Memory. Gija Local and Global Art Circulation, in Crossing Culture, Actes du Colloque du Comité International d'Histoire de l'Art, Melbourne, Melbourne University Press, pp. 823-828.

MORVAN, Arnaud, 2010, Traces en mouvement. Histoire, mémoire et rituel dans l'art kija contemporain du Kimberley Oriental (Nord-Ouest Australien), Thèse de doctorat, EHESS, Paris / University of Melbourne.

MunN, N., 1973, Warlbiri iconography, Ithaca, Cornell University Press.

MYERS, F. R., 2002, Painting culture. The making of an Aboriginal high art of Australia, Durham, Duke University Press,

PIERCE, C. S., 1955, Philosophical writings, New York, Dover, ed. J. Buchner.

RYAN, J. \& AKERMMAN, K. (eds), 1993, Images of power: Aboriginal art of the Kimberley, Melbourne, National Gallery of Victoria.

SEVERI, Carlo, 2007, Le principe de la Chimère. Une anthropologie de la mémoire, Paris, Édition Rue d'Ulm/Presses de l'ENS.

SEVERI, Carlo, 2003, Warburg anthropologue, ou le déchiffrement d'une utopie. De la biologie des images à l'anthropologie de la mémoire, L'Homme, 65, p. 77-129. 
STREHLOW, Theodor George Henry, 1971, Songs of Central Australia, Sydney, Angus \& Robertson.

TRELOYN, S., 2003, Scotty Martin's Jadmi Junba: A song series from the Kimberley region of northwest Australia, Oceania, vol. 73, no 3, pp. 208-220.

WALSH, M., 2007, Australian Aboriginal song language: So many questions, so little to work with, Australian Aboriginal Studies, 2, pp. 128-144.

WolfE, P., 1991, On being woken up: The dreamtime in anthropology and in Australian settler culture, Society and History, vol. 33, 2: 197-224.

\section{NOTES}

1. Cette étude se base sur plusieurs enquêtes de terrain réalisées dans la région du Kimberley oriental (Nord-Ouest de l'Australie) entre 2005 et 2008 et sur l'analyse d'un corpus d'une cinquantaine de peintures à l'ocre sur bois et sur toile réalisées entre 1979 et 2009 par huit artistes kija dont Rover Thomas et Paddy N. Bedford.

2. Plus de quatre-vingts ans après le début de la colonisation de la région, l'adoption du Federal Pastoral Award de 1969 par le gouvernement australien garantissait pour la première fois l'égalité salariale entre travailleurs blancs et aborigènes. Elle eut pour conséquence la réduction drastique du nombre d'employés aborigènes dans les fermes d'élevage et provoqua de larges déplacements de population vers les villes de Wyndham, Halls Creek, Fitzroy Crossing et Kununurra. Coupés de leurs terres, les Aborigènes étaient confrontés au chômage, à l'alcool et à la surpopulation. À l'époque, la nouvelle communauté de Warmun installée sur l'ancien relai télégraphique de Turkey Creek avait tout d'un camp de réfugiés. Après avoir longtemps travaillé sur la ferme de Texas Downs, Rover Thomas s'installa à Warmun en 1974.

3. En 1990 Rover Thomas est le premier artiste aborigène à représenter l'Australie à la Biennale d'Art contemporain de Venise. La même année il sera aussi exposé en France au musée Fabre de Montpellier et plusieurs grands musées australiens commencent à acquérir ses œuvres.

4. Paddy Nyunkuny Bedford est né au début des années 1920 à Bedford Down, une station d'élevage du Kimberley Oriental où il travaillera comme meneur de bétail pendant une grande partie de sa vie. En 1997, il rejoint la compagnie artistique Jirrawun Art et devient en quelques années l'un des peintres les plus reconnus du pays. Une rétrospective de ses œuvres a été organisée au Musée d'art contemporain de Sydney en 2006.

5. Une partie de cette cérémonie fut observée en 1938 par l'anthropologue allemand Andreas Lommel de la mission scientifique Frobenius, au cours d'un regroupement intertribal à plus de quatre cents kilomètres à l'ouest de Bedford Downs (Lommel, 1997 ; Morvan, 2010).

6. Les cérémonies publiques sont réalisées pour des occasions très variées : montrer la partie publique d'une cérémonie d'initiation masculine et féminine, rendre visibles certains aspects de la Loi pour les jeunes générations et/ou les étrangers, manifester des événements mythiques et/ ou historiques, résoudre un conflit à l'intérieur de la communauté ou avec d'autres groupes y compris non aborigènes, renouveler des alliances et des relations du wurnan (le réseau d'échanges rituels du Grand Nord-Ouest-australien).

7. C'est lors d'une enquête de terrain menée en 2007 que j'ai pu assister et participer à la conception d'une cérémonie joonba inédite nommée «Binyjrmin nawan » réalisée sur trois mois entre mai et juillet 2007. Le chant et les danses ont été créés par Phyllis Thomas en lien avec Paddy Bedford et Peggy Patrick, la cérémonie évoque sous forme métaphorique le parcours des artistes de Jirrawun, depuis la création des premières peintures dans l'outstation de Rugun, jusqu'à l'édification d'un atelier moderne (surnommé Binyjirmin Nawan « la grotte de la chauvesouris ») près de Wyndham, servant de base de travail et de lieu d'expositions. La cérémonie se 
présente comme l'exécution testamentaire de Paddy Bedford qui en est à l'origine et décéda quelques jours avant la fin de la préparation du joonba. Ce joonba avait alors pour but d'intégrer le nouvel atelier dans la cosmologie kija et dans le parcours historique des artistes (Morvan, 2010).

8. À plusieurs occasions pendant l'enquête, les créateurs du joonba ont localisé l'énergie du Ngarrangany (Dreaming) et des gamerre (scarifications rituelles) dans un "dessous " associé à l'intériorité du corps et de la terre: "we got our ngarrangarni from the ground ", expliquait Patrick. Le geste répété de martèlement du sol avec le pied semble être une volonté d'entrer en contact avec le ngarrangarni dans un double mouvement de creuser et lever, un peu comme un piston. En kriol, l'accès à un Dreaming est parfois désigné par le verbe "to pull out ", qui se traduit par «tirer » et « sortir ». Le geste des danseurs consiste donc à entrer en contact avec le dessous, d'accéder au ngarrangarni, compris comme une mémoire sacrée.

9. Transcription et traduction Frances Kofod et Arnaud Morvan (enregistrement de l'auteur).

10. Peggy Patrick, mai 2007, interview de l'auteur. Texte original: "Lumugul, that's our dreamtime, that's Neminuwarlin. That Lumugul been give us story,... to make story, that Binyjirmin and all. That because, he is from Crocodile Hole. He got the story for Crocodile Hole, for Rugun. We'll give you a story from (or for) Rugun through (or to) Naminuwarlin. That Naminuwarlin we call him Lumugul. 'Wad' means looking back, he looking back to that place, that Rugun. That the story (for) that joonba now." (...) "He looking back at us again".

11. Le chant suivant du joonba associe Rugun aux artistes et aux peintures: Ruguniyi, Ruguniyi, ngarag-garri yarremande, barlawandiyi, barlawandiyi. (At Crocodile Hole, at Crocodile hole we used to make things (the paintings) on the flat ground, on the flat ground.)

\section{RÉSUMÉS}

L'article propose un examen du rapport entre l'image et le chant dans la peinture kija du Kimberley Oriental (nord-ouest australien). Ces tableaux à l'ocre sur bois ou sur toile donnent à voir, sur le mode indiciaire, les traces laissées dans le paysage par des êtres totémiques, des esprits ou des personnages historiques. Ces traces sont chargées d'une triple mémoire mythique, historique et biographique inscrites dans un lieu. C'est en explorant les pratiques rituelles à l'origine de l'iconographie kija que l'article montre comment les chants induisent une mise en mouvement de l'image à la fois dans ces aspects esthétiques et structurels. Le chant agit ainsi comme un index dans un processus mnémonique d'association de lieux et d'événements dans une peinture.

The article investigates the links that take place between image and song within kija art traditions from the East Kimberley (north-west Australia). Kija ochre paintings show the tracks of totemic ancestors, spirits, or historical characters as they appear in the landscape. The tracks are charged with a triple memory of place, mythical, historical and biographical. An exploration of the ritual origins of kija iconography reveals the specific way songs generate movement in the aesthetical and structural components of the image. This movement is part of a larger mnemonic process of associating places and events in painting. 
INDEX

Index géographique : Australie

nomsmotscles Kija

Keywords : Australian Indigenous Art, Memory, Ritual, Song, Australia, Anthropology, Kija

Mots-clés : art aborigène australien, mémoire, rituel, chant

Thèmes : anthropologie (Océanie) 